\title{
Analytical Modeling and Experimental Validation of Electromagnetic Field Radiated by In-house PLC Lines
}

\author{
D. Chariag ${ }^{1}$, J-C. Le Bunetel ${ }^{1}$, K. Khalil ${ }^{1}$, Y. Raingeaud ${ }^{1}$, M. Machmoum ${ }^{2}$ and P. Guerin ${ }^{2}$ \\ 1. GREMAN: Research Group on Materials, Microelectronics, and Acoustic Nanotechnology, University of Tours, Tours 37200,
} France

2. IREENA: Institute of Research on Electrical Energy of Nantes-Atlantic, University of Nantes, Nantes 44300, France

\begin{abstract}
Broadband PLC (power line communication) technology is a main factor of the development of digital convergence in the indoor network. It uses the already existing power cable infrastructure for communication purposes. The EM (electromagnetic) field radiating from the cable could, however, disturb other communication systems, and thus should be evaluated. The MoM (method of moment) and the FEM (finite element method) have been studied to estimate the EM field emitted from the power cable. However, the MoM is difficult to treat the dielectric material of the cable and the FEM is time consuming. This paper presents a new approach to estimate the radiated EM fields caused by PLC sy stems from the CM current along the cable, based on the transmission line theory. The proposed model has the advantage of using the measured primary parameters of the cable. An experimental analysis of the EM radiation distribution is also presented. A comparison showed that the model results agree quite well with the measurements performed in this study.
\end{abstract}

Key words: PLC, EM field, transmission line theory, primary parameters.

\section{Introduction}

PLC (power line communication) technologies use the power line cable as the transmission line for both high data rate applications and control systems. The development of this technology is enhanced by the publication of the IEEE standard (IEEE Std 1901TM-2010) [1]. This standard provides a minimum implementation subset that allows a fair coexistence of the BPL (broadband over power line) devices. It also complies with EMC (electromagnetic compatibility) limits set by the national regulators.

EMC of PLC systems deals with two aspects. The first aspect is related to the susceptibility of the system in the presence of noise, and especially of impulsive noise [2]. This type of noise has been extensively characterized in the literature [3-10]. Zimmermann and Dostert classified the noise in PLC channels into

Corresponding author: Jean-Charles Le Bunetel, professor, research fields: transmission lines, communication power line, smart grid, EMC. five classes [3, 4]. A statistical model of the impulsive noise is proposed in Refs. [5, 6]. An estimation of the background noise is performed and presented in Ref. [7]. The periodic asynchronous impulsive noise is pointed out in Ref. [8]. In Ref. [9], the noise in narrowband PLC is expressed as a Gaussian process. In summary, there is a good understanding of the noise characteristics over indoor PLC channels. Nowadays, thanks to the optimization of modulation techniques and coding schemes, new PLC devices are generally able to deal with the different type of noises.

The second aspect of EMC of PLC systems is related to the emissions generated by these systems. For conducted emissions, the PLC signal is seen as a useful signal and there are few works dealing with the susceptibility of the household appliances to the PLC signal. However, numerous recent studies, which were carried out to analyze the coexistence between PLC systems and VDSL2 (very high bit-rate digital subscriber line) ones, showed that the radiated emissions from the PLC signal can lead to a decrease 
of the QoS (quality of service) of VDSL2 transmissions [11-15]. For example, it is indicated in Ref. [11] that the mutual impact of PLC and VDSL2 systems depends on the distance between electrical and phone lines, the length of the cables, the coexistence length, and the network imbalance.

In order to mitigate the radiated emissions from PLC systems, several studies have been made. The authors in Ref. [16-19] investigated the emission levels of existing PLC systems and compared them to the EMC standards such as FCC, EN55022 and NB30. Indeed, the radiation mainly depends on the $\mathrm{CM}$ (common mode) current [20]. This current is due to electrical asymmetries and to the LCL (longitudinal conversion loss) [20, 21]. An analysis of the DM (differential mode) to $\mathrm{CM}$ current conversion process in power line networks can be found in Ref. [22].

There has been a lot of theoretical modeling for radiated emissions from PLC systems [23-29]. Several methods have been used to compute the EM field such as the four-port network method [29], the MoM [28] and the so-called wire grid model [27].

Calculating the radiated field using the four-port network method cannot only be based on the CM current [28]. The method of moment cannot consider the influence of the dielectric material of the cable.

The so-called wire grid model (based on the electro-static theory [28]) computes the per-unit-length RLCG (resistance, inductance, capacitance, conductance). The RLC parameters are calculated from analytical expressions. The last parameter " $G$ " is deduced from measurements. The calculation results using these methods do not sufficiently agree with the measured ones. This paper proposes an appropriate calculation model for the radiated EM field from PLC lines. Firstly, the RLCG parameters of electrical cable considering the ground wire are extracted from measurements. Then, these parameters are used by the SPICE simulator to calculate the CM current distribution along the cable. Finally, the EM field is deducted using infinitesimal dipole model [30]. The presented model is validated with measurements using commercial PLC modems.

The remainder of this paper is organized as follows: in Section II, a detailed description of the measurement set-up is given. The HF (high frequency) model of the considered network is described in Section III. The mathematical model of the radiated EM field is presented in Section IV. Section V provides a comparison between the simulated EM fields and the experimental ones. Finally, Section VI concludes this paper.

\section{Measurement Set up}

The radiated EM field was measured using the experimental set-up as shown in Fig. 1. The measurement was carried out in an anechoic chamber of length and width equal to $9.2 \mathrm{~m}$ and $7.6 \mathrm{~m}$, respectively. The measured frequency range was from $0.15 \mathrm{MHz}$ to $30 \mathrm{MHz}$. The electric and magnetic fields were measured separately using monopole and loop antennas. This allows near-field measurements. The height of the antenna is $1.7 \mathrm{~m}$ and the horizontal distance from the electrical cable is $1.0 \mathrm{~m}$. The antenna output voltage was measured using a spectral analyzer. The analyzer was installed outside the anechoic chamber and was connected to the antenna using a coaxial cable.

Our purpose is to replicate the real operating conditions of a PLC connection in the semi-anechoic chamber. To this end, the PLC signal was injected using two HPAV (Home Plug AV) modems (offers a physical layer peak data rate of $200 \mathrm{Mbits} / \mathrm{s}$ ). In the indoor PLC network, the transmitter (PLC modem) is connected on one side to an ADSL (asymmetric digital subscriber line) modem using the Ethernet cable and on the other side to the electrical grid. To replace the ADSL connection, the ADSL modem is connected to a video server using a DSLAM (digital subscriber line access multiplexer). A category 5 cable is used between the DSLAM and the ADSL modem. 


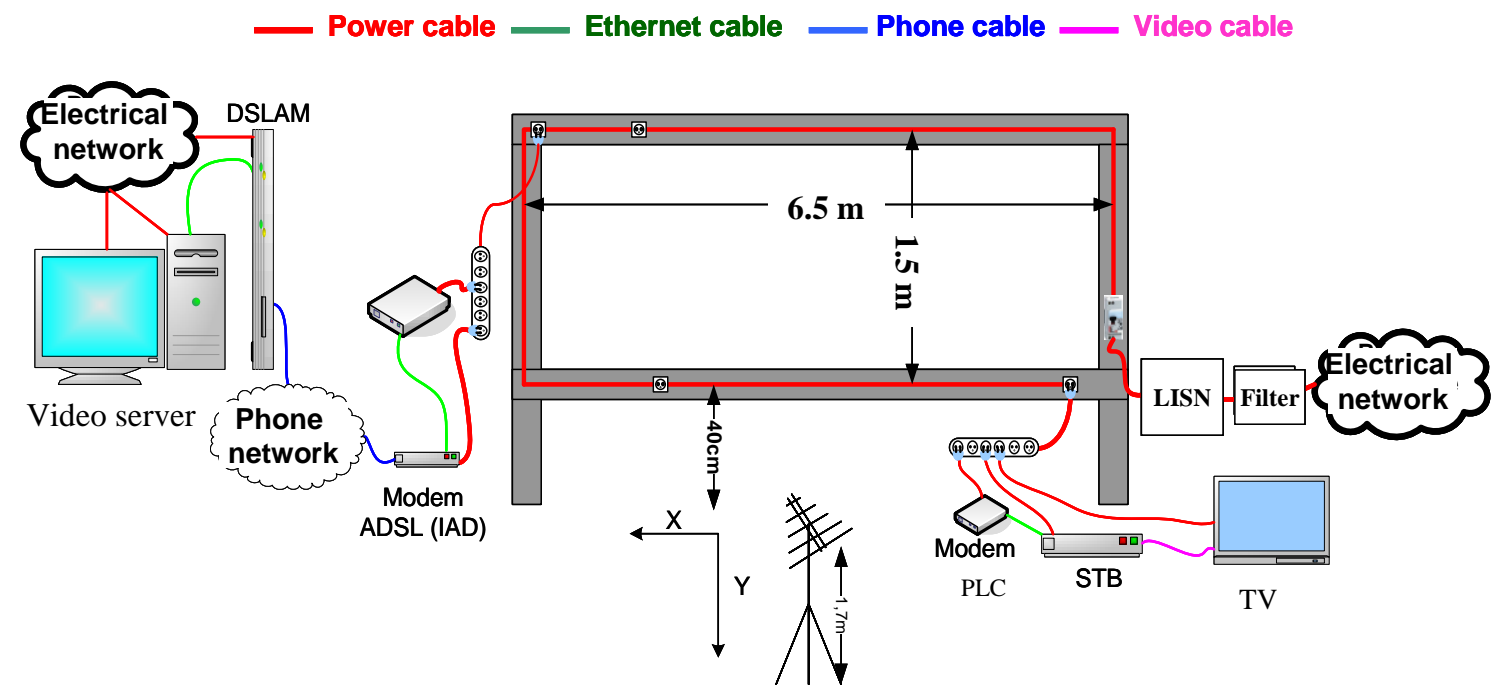

Fig. 1 The experimental set-up.

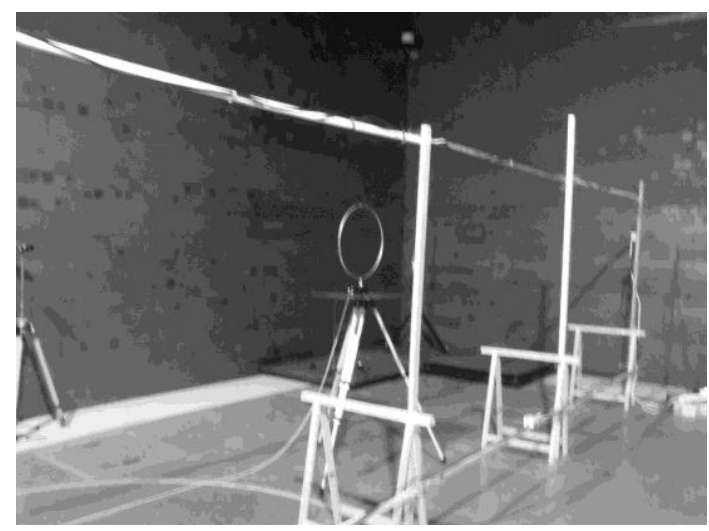

Fig. 2 A photo of the experimental grid.

Fig. 1 depicts the experimental network. The electrical cable is attached to a wooden support forming a rectangular loop at a height of $0.4 \mathrm{~m}$ from the ground level (Fig. 2).

The horizontal and vertical lengths of the loop are respectively $1.5 \mathrm{~m}$ and $6.5 \mathrm{~m}$, so the total length is $16.0 \mathrm{~m}$. The power cable is made up of three wires (phase, neutral and ground) coated in insulating PVC. Adding a ground wire is important to take into account the propagation noise in the $\mathrm{CM}$ signal. The cross-section area of the wire is $2.5 \mathrm{~mm}^{2}$. The ADSL modem and the transmitter PLC modem are plugged into the electrical network through the same multi-plug. The receiver PLC modem, the TV and the video box are connected to the grid using a second multi-plug as shown in Fig. 1.
The presented network is connected to the power grid through an LISN (line impedance stabilizer network), a low pass filter and an isolation transformer. This reduces the EM noise from the power netw ork.

\section{High Frequency Model of the Power Network}

The distribution of the CM current should be calculated to estimate the radiated EM field. In this paper, the CM current distribution was simulated using the LTspice software. The simulation was carried out using the RLCG parameters of the power cable, the LC (inductance and capacitance) model of the multi-plug and the measured impedances of the connected devices (TV, video box, modems). This section details the model of these components (power cable, multi-plug, connected devises).

\subsection{Power Cable Model}

The power cables can be described and modeled using the transmission line theory. In this theory, the power cable is considered as a series of elementary cells, each representing a short segment of the transmission line. As shown in Fig. 3, every cell can be described by the so-called primary line parameters, usually denoted as RLCG. The type of the power 


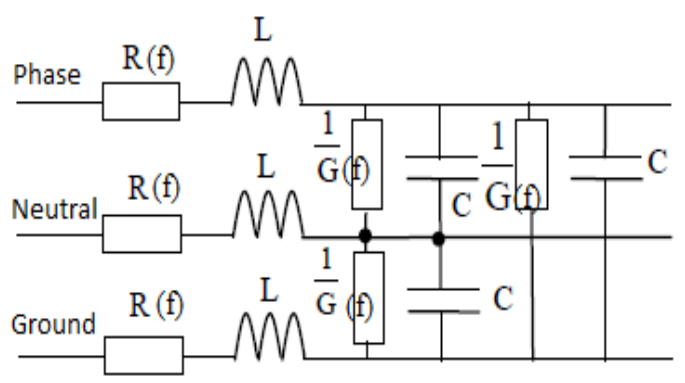

Fig. 3 Model of a three-conductor cable with the primary parameters.

cable concerned by the present study is RVV 3 G 2.5 $\mathrm{mm}^{2}$. The length of the elementary cell is equal to $25.0 \mathrm{~cm}$ in the 1 to $50 \mathrm{MHz}$ frequency range. It should be noted that the length of the elementary segment must be chosen as a function of the studied frequency range. Indeed, this length should be negligible compared to the shortest wavelength; for a maximum operating frequency of $50 \mathrm{MHz}$, the shortest wavelength is about $4.0 \mathrm{~m}$.

There are several methods to estimate the value of the primary parameters, based on analytical expressions, finite element simulation or experimental measurements [31]. In this paper, the RLCG parameters are extracted from measurements in the 1 to $50 \mathrm{MHz}$ frequency range. The measurement process is fully described in Ref. [31]. Next, an electrical circuit is assigned for each parameter (R(f)-L-C-G(f)) as shown in Fig. 4. In order to improve the model accuracy, the value of each electrical component is realized using genetic algorithms [32].

\subsection{HF Model of the Multi-plug}

The multi-plug comprises two parts: the exterior part which is the cable and the interior one which includes two bus-bar, as shown in Fig. 5. The primary RLCG parameter of a $25.0 \mathrm{~cm}$ cell is deduced from open and short measurements method described in Ref. [31].

The bus-bar is divided into segments of $10 \mathrm{~cm}$ as shown in Fig. 5. The open and short measurements method was applied to calculate the primary parameters of the bus-bar segment. The $R$ and $G$ parameters are not considered in modeling the bus-bar. Indeed, the copper and the dielectric losses of the considered bus-bar are very small. The measurements show that the values of $\mathrm{L}$ and $\mathrm{C}$ parameters are constant for all measured frequencies $(\mathrm{L}=17.7 \mathrm{nH}$ and $\mathrm{C}=2.65 \mathrm{pF}$ ). Fig. 6 shows the circuit model of the multi-plug (includes 5 plugs).
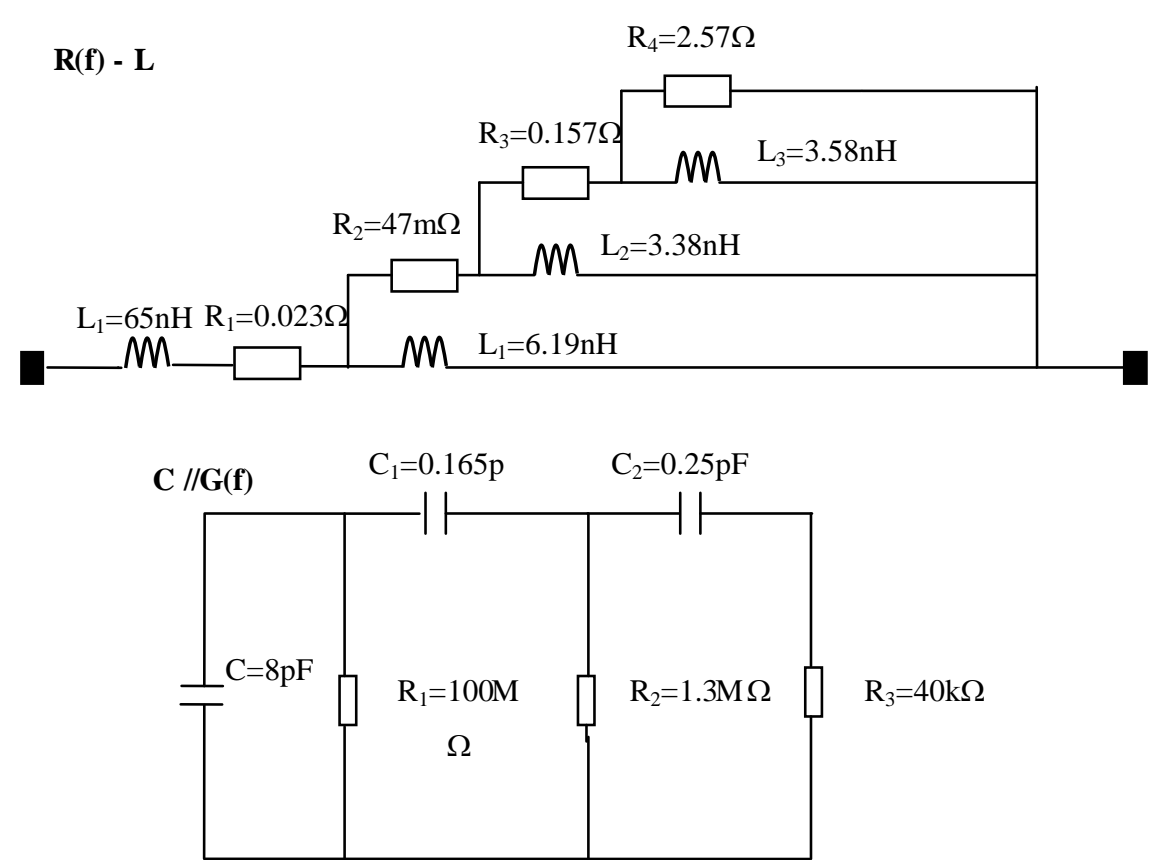

Fig. 4 Equivalent circuit of primary parameters RLCG. 

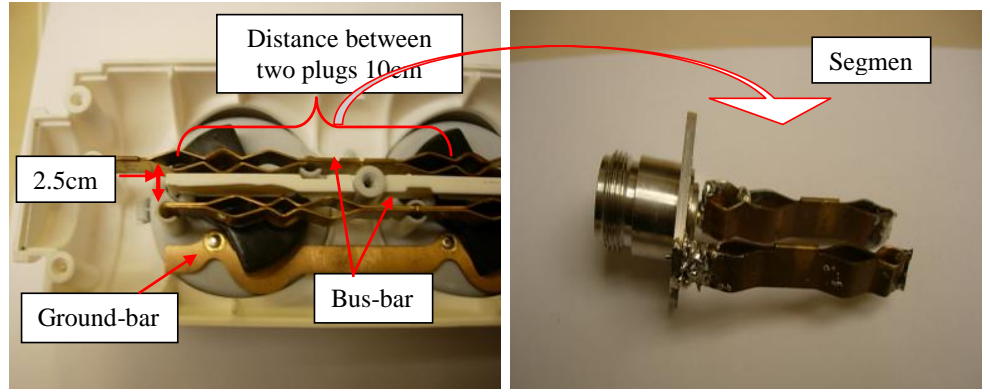

Fig. 5 Photo of the multi-plug bus-bar.
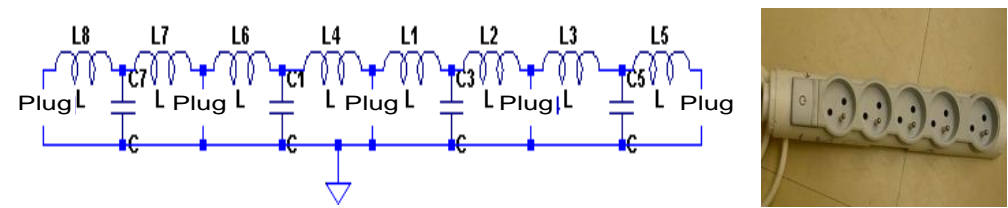

Fig. 6 Model of the bus-bar.

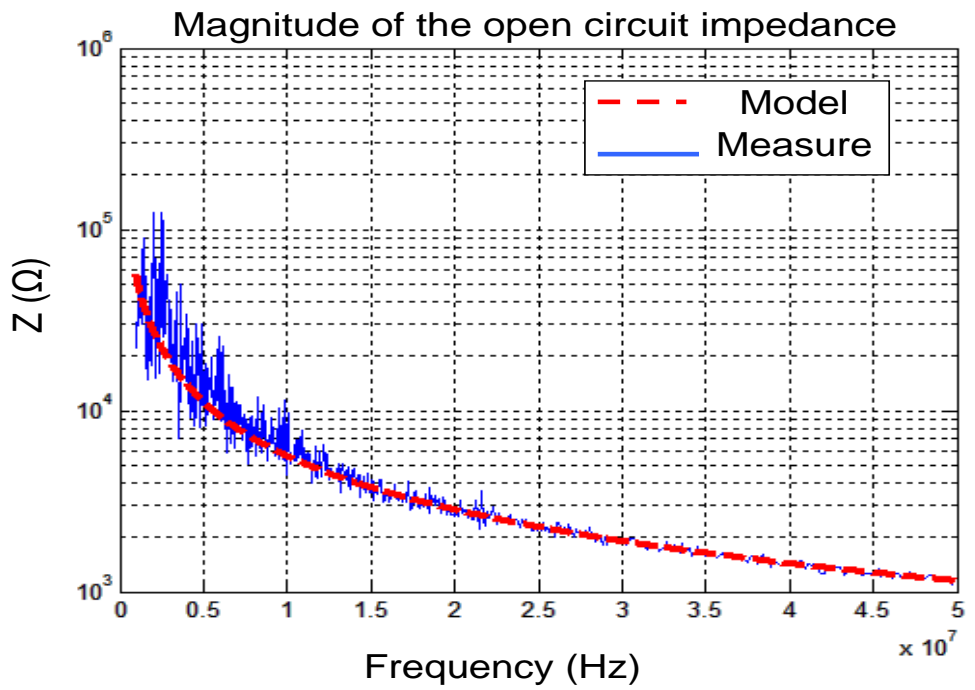

Fig. 7 Comparison between measured and modeled impedances of the bus-bar.

Fig. 7 shows a comparison between measured and modeled impedances of the bus-bar. A good agreement between the two plots can be seen. For the sake of simplicity, the ground bus is not considered in the model.

The experimental network comprises two multi-plugs. Both the ADSL modem and transmitter PLC modem are connected to the first multi-plug. The TV and the video box are connected to the second one. These devices are modeled using the measured impedances.

\subsection{HF Model of the Connected Appliances}

The appliances connected to the experimental network (TV, ADSL modem, video box and the LISN) are modeled using their measured impedances. In fact, an arbitrary voltage source in the LTspice software is controlled using a mathematical expression or a data file. In this work, a measurement database file is used to control the arbitrary voltage source.

The measurement set-up of the household appliances is described in Ref. [33] and depicted in Fig. 8.

\subsection{Experimental Validation of the Modeled Network}

The network model (shown in Fig. 1) is built using the LTspice software as shown in Fig. 9. The power cable is modeled using the primary RLCG parameters. The multi-plug is modeled using the primary parameters 


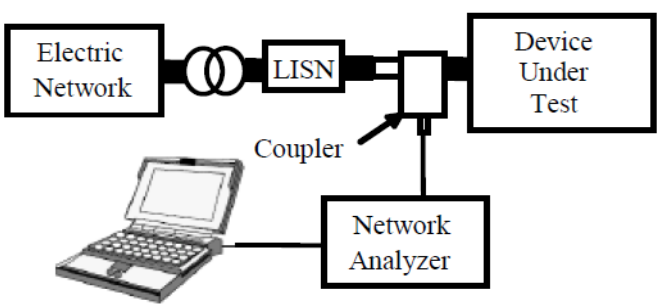

Fig. 8 Impedance measurement test bench.

described previously. The measured impedances of the connected devices are integrated in the simulation file.

To validate the frequency model of the network, the transmission parameter $\mathrm{S} 21$ between two points is measured and compared to the simulation. The results are presented in Fig. 10. A similar trend is observed between the simulated and the measured transmission parameter. However, a deviation of $5 \mathrm{~dB}$ is noted around the frequency of $22 \mathrm{MHz}$. A low deviation is also detected around $35 \mathrm{MHz}$.

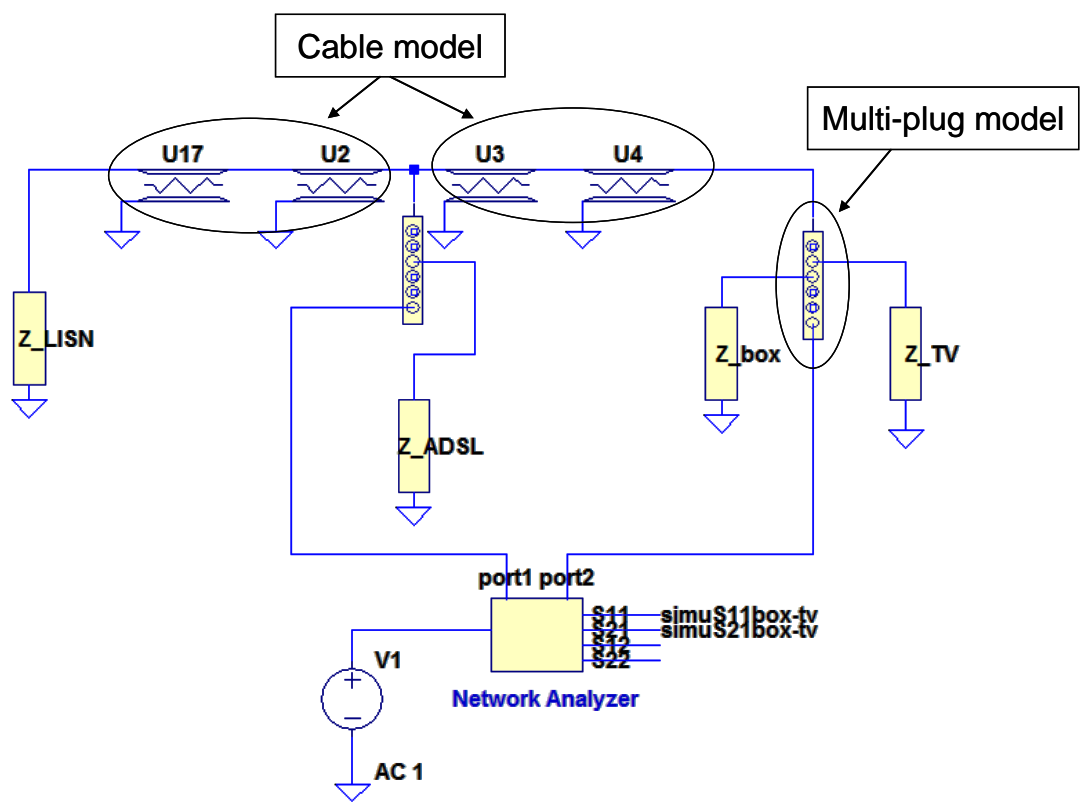

Fig. 9 LTspice model of the experimental network.

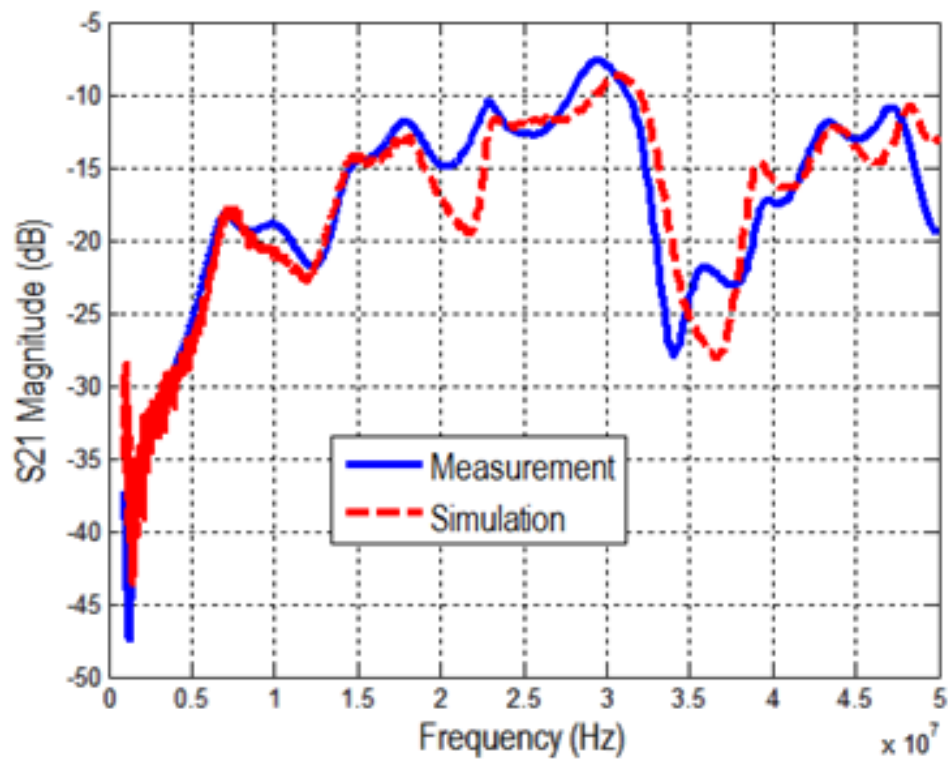

Fig. 10 Comparison between measured and simulated $\mathrm{S} 21$ parameter. 


\section{Analysis of the Radiated EM Fields}

As described in the literature, the PLC radiation is mainly depending on the CM current [20]. So, the elementary cell of the three-conductor cable acts as a thin wire (very small radius compared to the length) and the assumption of infinites imal dipole can be used. In fact, the length of the elementary cell $(25 \mathrm{~cm})$ is much smaller than the shortest wavelength up to 30 MHz. Assuming that the current is located at the $z$-axis $\left(\vec{I}=I_{0} \vec{u}_{z}\right)$, and the distance of the observation point $(\mathrm{M}(r, \theta, \varphi))$ from the origin is $r$, as shown in Fig. 11. The vector potential in spherical coordinates is given by Eqs. (1) and (2), where $\mu_{0}$ is the permeability of vacuum and $l=d z$ is the length of the dipole. $A_{\varphi}$ is zero when the current is located at the $z$-axis. The magnetic field is calculated through Eqs. (1-3).

$$
\begin{gathered}
A_{r}=\mu_{0} I_{0} l \cos (\theta) \frac{e^{-j k r}}{4 \pi r} \\
A_{\theta}=-\mu_{0} I_{0} l \sin (\theta) \frac{e^{-j k r}}{4 \pi r} \\
\vec{H}=\frac{1}{\mu_{0}} \nabla \times \vec{A}=\frac{1}{\mu_{0} r}\left[\frac{\partial}{\partial r}\left(r A_{\theta}\right)-\frac{\partial A_{r}}{\partial \theta}\right] \overrightarrow{u_{\varphi}}
\end{gathered}
$$

Substituting Eqs. (1) and (2) in Eq. (3) reduces the magnetic field to:

$$
H_{\varphi}=j \frac{k I_{0} l \sin (\theta)}{4 \pi r}\left[1+\frac{1}{j k r}\right] e^{-j k r}, H_{\theta}=H_{r}=0
$$

The electric field is obtained from the vector potential Eqs. (1) and (2) using Eq. (5).

$$
\vec{E}=-j \omega \vec{A}-j \frac{1}{\omega \mu_{0} \varepsilon_{0}} \nabla(\nabla \vec{A})=\frac{1}{\omega \mu_{0} \varepsilon_{0}} \nabla \times \vec{H}
$$

In spherical coordinates, we have:

$$
\left\{\begin{array}{l}
E_{r}=\eta \frac{I_{0} l \cos (\theta)}{2 \pi r^{2}}\left[1+\frac{1}{j k r}\right] e^{-j k r} \\
E_{\theta}=j \eta \frac{k I_{0} l \sin (\theta)}{4 \pi r}\left[1+\frac{1}{j k r}-\frac{1}{(k r)^{2}}\right] e^{-j k r} \\
E_{\varphi}=0
\end{array}\right.
$$

where $\eta$ is the impedance of free space $(\eta=120 \pi)$.
The magnetic field in Eq. (4) and the electric field in Eq. (6) are valid everywhere except on the source itself (cable). The EM fields vary according to the distance $r$ between the dipole and the observation point. Depending on this distance, three regions are identified: the near field region ( $\mathrm{k} . \mathrm{r}<1)$, the middle region (k. $r \geq 1)$ and the far field region (k. $r>>1)$.

To evaluate the EM fields radiated from the netw ork (depicted in Fig. 1), the circuit model (Fig. 9) was simulated using the LTspice software. The PLC modem is modeled using a $50 \Omega$ impedance in parallel with an AC voltage source (port 1). For each elementary cell, the simulated CM current was used to calculate the EM fields according to Fig. 12. Eqs. (4)

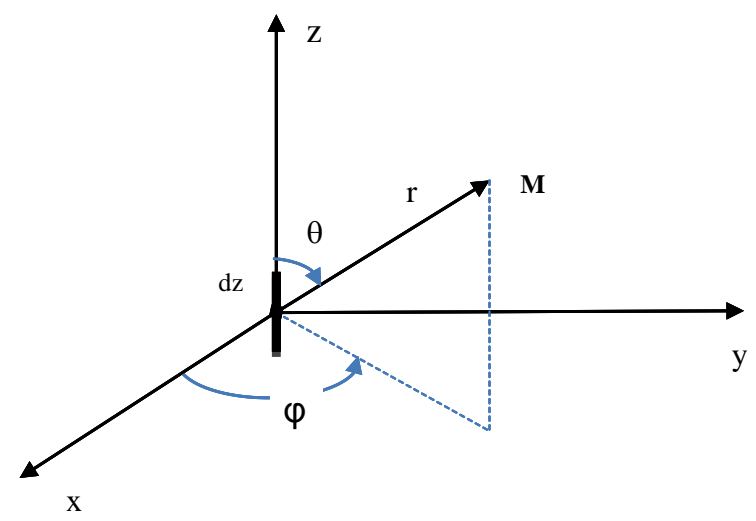

Fig. 11 Infinitesimal dipole.

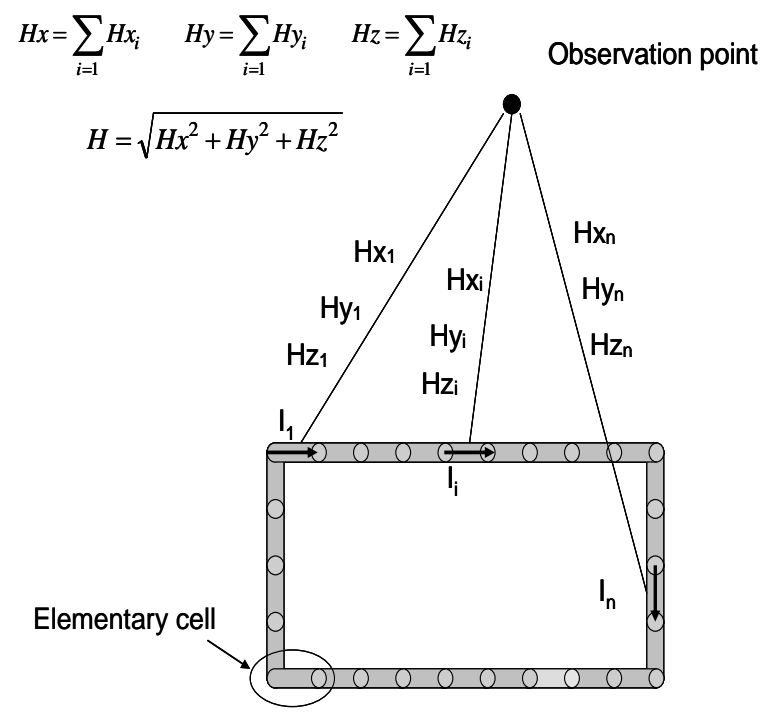

Fig. 12 Analysis model of radiating magnetic field. 
and (6) are transferred to Cartesian coordinates. The total field is the vector sum of the fields radiated from each elementary cell.

\section{Comparison between the Model and the Measurement}

Fig. 13 shows the comparison between the measured magnetic field and the calculated one using the proposed model. Two regions are identified from this comparison. The first region includes two frequency bands from $150 \mathrm{kHz}$ to $2 \mathrm{MHz}$ and from 10 $\mathrm{MHz}$ to $13 \mathrm{MHz}$. A large gap is noted between the model and the measurement for these frequency bands. For the $150 \mathrm{kHz}$ to $2 \mathrm{MHz}$ band, these errors can be justified. Indeed, the background noise and the noise generated by the connected devices are not taken into account in the model. The second region includes frequencies from $2 \mathrm{MHz}$ to $10 \mathrm{MHz}$ and from $13 \mathrm{MHz}$ to $30 \mathrm{MHz}$. A good agreement can be noted between measurement and model results for this region.

Fig. 14 shows the measured and calculated electric field distributions. The measured values agreed quite

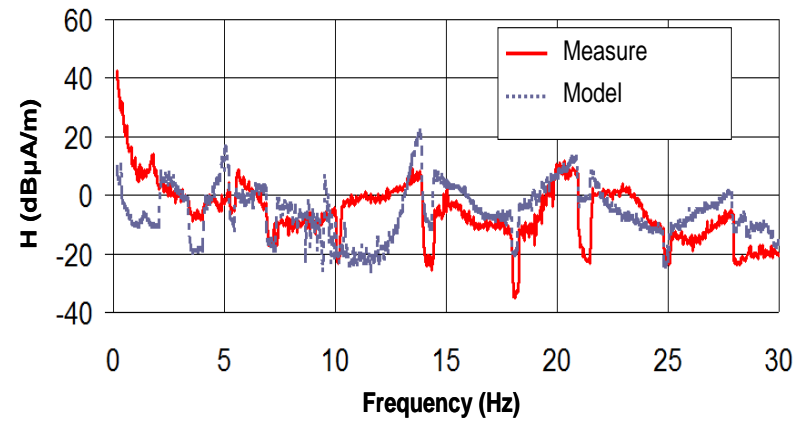

Fig. 13 Radiated magnetic field.

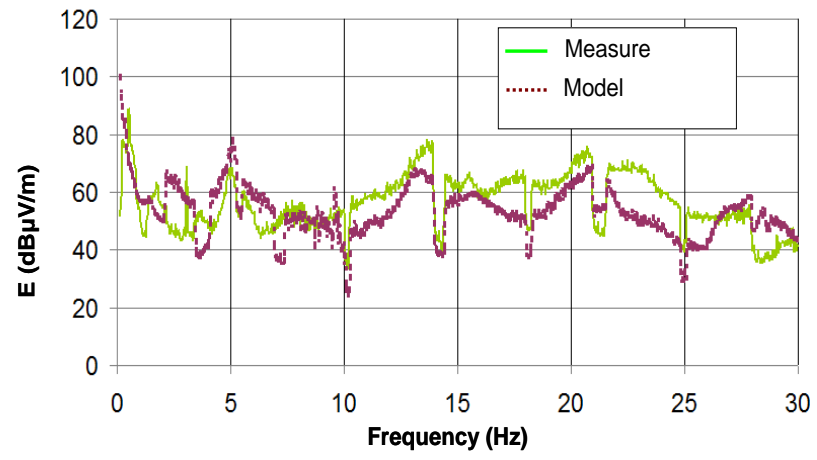

Fig. 14 Radiated electric field. well with that of the calculated ones for all frequencies.

\section{Conclusion}

In this paper, we addressed the issue of radiated emissions caused by in-house PLC systems based on experimental measurements. The measurements were performed inside an anechoic chamber in a frequency band up to $30 \mathrm{MHz}$. A new approach to estimate the radiated EM fields from PLC systems using the transmission line theory was provided. The proposed model allows the calculation of the radiated EM field components from the $\mathrm{CM}$ current along the cable by considering the ground conductor. The model has the advantage of using the primary parameters extracted from the measurement. This allows to reduce the modeling errors compared to other methods such as analytical and numerical calculations ones. The comparison between experiment and model results showed that the model can provide good EM interference predictions in PLC.

The aim of future studies is to extend the model up to $100 \mathrm{MHz}$. Other type of cable could also be modeled.

\section{Ackno wledge ments}

This research was supported by the French Region Center with the MDE MAC3 project funding.

\section{References}

[1] IEEE Standard for Broadband over Power Line Networks: Medium Access Control and Physical Layer Specifications, IEEE Std 1901'TM-2010.

[2] Degauque, P., Laly, P., Degardin, V., Lienard, M., and Diquelou, L. 2010. "Compromising Electromagnetic Field Radiated by In-house PLC Lines." In Proceedings of the Global Communications Conference.

[3] Zimmermann, M., and Dostert, K. 2000. "An Analy sis of the Broadband Noise Scenario in Powerline Networks." In Proceedings of the 4th International Symposium on Power-Line Communications and Its Applications.

[4] Zimmermann, M., and Dostert, K. 2002. "Analysis and Modeling of Impulsive Noise in Broad-Band Powerline Communications." IEEE Transactions on 
Electromagnetic Compatibility 44 (1): 249-58.

[5] Meng, H., Guan, Y. L., and Chen, S. 2005. "Modeling and Analy sis of Noise Effects on Broadband Power-Line Communications." IEEE Transactions on Power Delivery 20 (2): 630-7.

[6] Rouissi, F. 2008. "Optimisation de la couche PHY des systèmes de communication sur le réseau d'énergie en présence de bruit impulsif." Ph.D. thesis, SUP'COM, Tunis.

[7] Guillet, V., and Lamarque, G. 2010. "Unified Background Noise Model for Power Line Communication." IEEE International Symposium on Power Line Communications.

[8] Cortés, J. A., Diez, L., Canete, F. J., and Lopez, J. 2009. "Analysis of the Periodic Impulsive Noise Asynchronous with the Mains in Indoor PLC Channels." IEEE International Symposium on Power Line Communications.

[9] Katayama, M., Yamazato, T., and Okada, H. 2006. "A Mathematical Model of Noise in Narrowband Power Line Communication Systems." IEEE Journal on Selected Areas in Communications 24 (7).

[10] Chariag, D., Guezgouz, D., Bunetel, J-C. Le, and Raingeaud, Y. 2012. "Modeling and Simulation of Temporal Variation of Channel and Noise in Indoor Power Line Network." IEEE Transactions on Power Delivery 27 (4).

[11] Moulin, F., Péron, P., and Zeddam, A. 2010. "PLC and VDSL2 Coexistence." IEEE International Symposium on Power Line Communications.

[12] Praho, B., Tlich, M., Zeddam, A., Moulin, F., and Nouvel, F. 2012. "A PLC/VDSL2 Coexistence Method Based on Noise Injection on Powerline during VDSL2 Initialization." International Symposium on Electromagnetic Compatibility.

[13] Kerpez, K. et al. 2015. "The Impact of PLC-to-DSL Interference on VDSL2, Vectored VDSL2, and G.fast." IEEE International Symposium on Power Line Communications.

[14] Praho, B., Razafferson, R., Tlich, M., Zeddam, A., and Nouvel, F. 2011. "Study of the Coexistence of VDSL2 and PLC by Analy sing the Coupling between Power Line and Telecommunications Cable in the Home Network." 30th General Assembly and Scientific Symposium.

[15] Praho, B., Zeddam, A., Moulin, F., and Nouvel, F. 2011. "A Cognitive Method for Ensuring the Coexistence between PLC and VDSL2 Technologies in Home Network." In Proceeding of the IEEE International Conference on Microwaves, Communications, Antennas and Electronic Systems.

[16] Adebisi, B., and Honary, B. 2006. "Comparisons of Indoor PLC Emissions Measurement Results and
Regulation Standards." IEEE International Symposium on Power Line Communications, 319-24.

[17] Zeddam, A., Razafferson, R., Gauthier, F., and Pagani, P. 2008. "Analysis of EMC Issues and Throughputs of the PLC Systems up to $100 \mathrm{MHz}$." International Union of Radio Science General Assembly.

[18] Vick, R. 2001. "Radiated Emission Caused by In-house PLC-Sy stems." IEEE International Symposium on Power Line Communications.

[19] Marthe, E., Rachidi, F., and Ianoz, M. 2003. "Evaluation of Indoor PLC Radiation Resulting from Conducted Emission Limits." IEEE International Symposium on Electro Magnetic Compatibility.

[20] Vukicevic, A. 2008. "Electromagnetic Compatibility of Power Line Communication Systems." Ph.D. thesis, École Poly technique Fédérale de Lausanne.

[21] Favre, P., Candolfi, C., Schneider, M., Rubinstein, M., Krähenbuehl, P., and Vukicevic, A. 2007. "Common Mode Current and Radiations Mechanisms in PLC Networks." IEEE International Symposium on Power Line Communications.

[22] Vukicevic, A., Rubinstein, M., Rachidi, F., and Bermudez, J. L. 2006. "On the Mechanisms of Differential-Mode to Common-Mode Conversion in the Broadband over Power Line Frequency Band." 17th International Zurich Symposium on Electromagnetic Compatibility.

[23] Naiman, S., Kissaka, M. M., Hamad, O. F., and Anatory, J. 2014. "Prominent Players of EM Field Radiation and Emission in BPLC Line." International Journal of Renewable Energy Technology 3 (5): 699-702.

[24] Chaaban, M., Drissi, K. El K., and Poljak, D. 2012. "Analytical Model for Electromagnetic Radiation by Bare-Wire Structures." Progress in Electromagnetics Research B 45: 395-413.

[25] Chaaban, M., Bousaleh, G., Chehade, R. H., Ismail, A., Drissi K. El K., and Pasquier, C. 2009. "Reduction of Power Field Radiation for PLC Applications." In Proceedings of the IEEE International Conference on Advances in Computational Tools for Engineering Applications.

[26] Luo, and Tan, S. Y. 2006. "A Radiated Emission Model for Power-Line Communications." IEEE Transactions on Power Delivery 21 (3).

[27] Nagamoto, K., Kuwabara, N., and Kawabata, M. 2008. "Calculation of Emitted Magnetic Field Using Wire Grid Model Considering Dielectric Material of VVF Cable." EMC Europe.

[28] Kojima, N., Kawabata, M., and Kuwabara, N. 2009. "Calculation of Radiated Electromagnetic Field from Multi-Pair Cable by Method of Moment." International Symposium on Electromagnetic Compatibility. 

Radiated by In-house PLC Lines

[29] Miyoshi, K., Kuwabara, N., Akiyama, Y., and Yamane, H. 2005. "Calculation of Radiating Magnetic Field from Indoor AC Mains Cable Using Four-Port Network." International Symposium on Electromagnetic Compatibility.

[30] Balanis, C. A., ed. 1996. Antenna Theory: Analysis and Design. Wiley-Interscience.

[31] Chariag, D., Guez gouz, D., Raingeaud, Y., and Bunetel, J-C. Le. 2011. "Channel Modeling and Periodic Impulsive Noise Analysis in Indoor Power Line." IEEE
International Symposium on Power Line Communications.

[32] Goldberg, D. E. 1989. Genetic Algorithms in Search, Optimization, and Machine Learning. Addison-Wiley Publishing Company.

[33] Chariag, D., Bunetel, J-C. Le, and Raingeaud, Y. 2012. "A Method to Construct Equivalent Circuit from Input Impedance of Household-Appliances." International Journal on Communications Antenna and Propagation 2 (4): 226. 